\title{
A Computational Investigation of an Integro-Differential Inequality with Periodic Potential
}

\author{
B.M. BROWNa and V.G. KIRBY ${ }^{b, *}$ \\ a Department of Computer Science, Cardiff University, P.O. Box 916, \\ Cardiff CF3 3 XF, UK; ${ }^{b}$ School of Mathematics, Kingston University, \\ Penrhyn Road, Kingston Upon Thames, Surrey KT1 2EE, UK
}

(Received 16 April 1999; Revised 12 July 1999)

This paper is concerned with a certain integral inequality whose associated differential expression contains a periodic potential. In these problems the spectrum consists of bands which may have eigenvalues in the gaps. We use numerical methods to estimate the location of these bands and position of the eigenvalues and use this information to produce new examples of the HELP inequality.

Keywords: Integral inequality; Ordinary differential equations;

Titchmarsh-Weyl $m$-function; Periodic potential; Numerical methods

1991 AMS Mathematics Subject Classifications: 34, 65

\section{INTRODUCTION}

This paper is concerned with the study of an integral inequality which involves the second order Sturm-Liouville differential expression

$$
M[f]:=-f^{\prime \prime}+q(x) f
$$

over $[a, b)$. Here $f$ is some function in the set $\Delta$ (see below), and $q$ is locally integrable and periodic with period $2 \pi$, thus $q$ satisfies minimal

\footnotetext{
* Corresponding author.
} 
conditions for the equation $M[f]=\lambda f(\lambda \in \mathbf{C})$ to be regular at 0 . The inequality that we study is

$$
\left(\int_{0}^{\infty}\left(\left|f^{\prime}\right|^{2}+q|f|\right) \mathrm{d} x\right)^{2} \leq K \int_{0}^{\infty}|f| \mathrm{d} x \int_{0}^{\infty} M[f] \mathrm{d} x,
$$

where the inequality is required to hold, with finite constant $K$, for all members $f$ of the set

$$
\Delta \equiv\left\{f: f, f^{\prime} \in A C_{\mathrm{loc}}[a, b) ; f, M[f] \in L^{2}[a, b)\right\} .
$$

In the above $A C_{\text {loc }}[a, b)$ is the set of functions which are locally absolutely continuous on the interval $(a, b)$ and $L^{2}(a, b)$ denotes those functions $f$ which belong to the Lebesgue square integrable space over $(a, b)$. The inequality (1.2) is an instance of Everitt's HELP inequality, this being a development of a classical inequality, the case $q=0$, due to Hardy and Littlewood. A feature of Everitt's treatment of (1.2) in [12] and Evans and Everitt's analysis in [9] is that the existence of the inequality together with the best constant is determined by the behaviour of the Titchmarsh-Weyl $m$-function associated with the differential expression (1.1).

When $q(x)=x^{\alpha}-\tau, \alpha>0$ and $\tau$ a real constant the inequality (1.2) has been the subject of much study. In the special case $q(x)=-\tau$ the inequality is valid if and only if $\tau \geq 0$ in which case $K=4$. When $\alpha>0$ it is known that a valid inequality can occur if and only if $\tau$ is either a Neumann or Dirichlet eigenvalue of (1.1) and analytic results on the existence of and value of the best constant $K=K(\alpha)$ are known only in the special cases $\alpha=1,2$ (see [10] for a fuller discussion). An extensive numerical investigation of the inequality for $0 \leq \alpha \leq 10$ has shown that when $\tau$ is a Neumann translate (that is we have translated the problem (1.1) by an eigenvalue with Neumann boundary conditions imposed at the regular end-point 0 ) the inequality is valid for all $\alpha$ with $K=4$ (see [14]). When $\tau$ is a Dirichlet translate the inequality is again still valid for all $\alpha$ with the best constant taking values depicted in Fig. 1.

An important difference between the situation when $q(x)=x^{\alpha}-\tau$ and when $q(x)=-x^{\alpha}$ is that in the former case the spectrum of (1.1) is discrete, consisting only of eigenvalues, while in the latter it consists of pure continuous spectrum over $(-\infty, \infty)$ and thus the inequality may be valid for all translates. In this paper we shall be concerned with an 


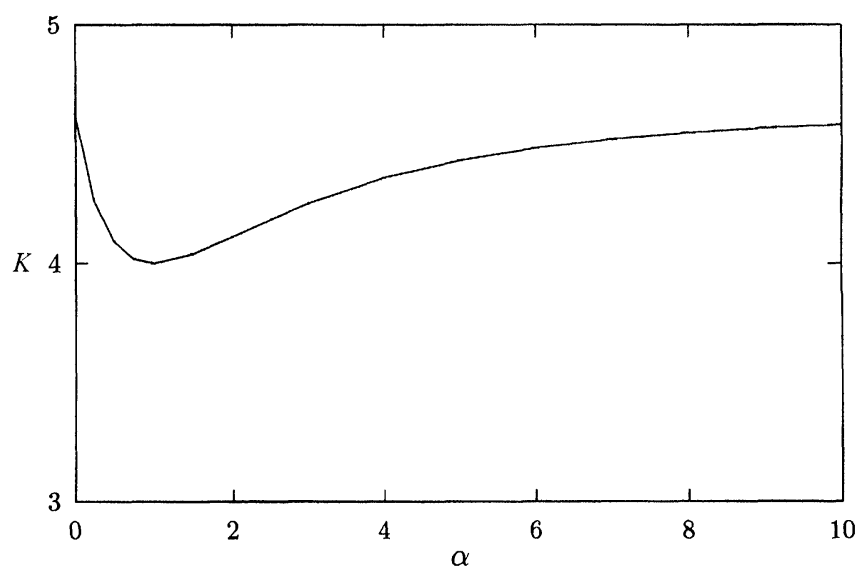

FIGURE 1 Best constants $K$ plotted against $\alpha$ for $q(x)=x^{\alpha}-\tau_{1}(D)$, first eigenvalue considered with Dirichlet boundary conditions.

investigation of the inequality (1.2) when $q(x)$ is $\pm \sin x, \pm \cos x$ and

$$
q(x)= \begin{cases}-1, & x \text { on }[0, \pi] \\ +1, & x \text { on }[\pi, 2 \pi]\end{cases}
$$

and then extended by periodicity over the interval $[0, \infty)$.

A feature of these examples is that the continuous spectrum of (1.1) lies in bands with any eigenvalues confined to the spectral gaps. We shall provide strong numerical evidence that the inequality is valid when zero lies in one of the spectral bands and also when zero is a Neumann or Dirichlet eigenvalue. That is, we translate the problem (1.1) by a value equal to the eigenvalue. In later sections we compute the position of these bands using the Rayleigh-Ritz method and examine various translates to show how the values of the best constants vary.

\section{PRELIMINARIES}

The most general formulation of Everitt's extension to the HardyLittlewood inequality is given in Evans and Everitt [9]. To be specific we shall need the following notions from the spectral theory of ordinary differential equations. 
We say that (1.1) is limit-point (LP) at the singular point $b$ if there is precisely one solution of (1.1) in $L^{2}[a, b)$ (up to a constant multiple) and this is equivalent to the condition that $\lim _{x \rightarrow b_{-}} p\left(f \bar{g}^{\prime}-f^{\prime} \bar{g}\right)(x)=0$ for all $f, g \in \Delta$. If the stronger condition $\lim _{x \rightarrow b_{-}} p\left(f \bar{g}^{\prime}\right)(x)=0$ holds for all $f, g \in \Delta$ then we say that (1.1) is strong limit-point (SLP) at $b$. Clearly SLP implies LP; it is known that the converse is not true.

If $M[f]$ is SLP at $b$, then for all $f \in \Delta$, it may be shown using the Dirichlet formula for (1.1) that the left hand side of (1.2) exists, but the integral may not be absolutely convergent. If (1.1) is LP at $b$ and regular at $a$ then there exists a pair of functions $m_{+}$and $m_{-}$defined on the upper and lower half-planes $\mathbf{C}_{+}, \mathbf{C}_{-}$of the complex plane $\mathbf{C}$ respectively, which possess the following properties:

(i) $m_{ \pm}: \mathbf{C}_{ \pm} \rightarrow \mathbf{C}_{ \pm}$;

(ii) $m_{ \pm}$is analytic on $\mathbf{C}_{ \pm}$;

(iii) $\bar{m}_{ \pm}(\lambda)=m_{\mp}(\bar{\lambda})\left(\lambda \in \mathbf{C}_{ \pm}\right)$;

(iv) let $\theta, \phi$ be solutions of (1.1) that satisfy

$$
\theta(a)=0, \quad \theta^{\prime}(a)=1 ; \quad \phi(a)=-1, \quad \phi^{\prime}(a)=0
$$

and

$\psi(x, \lambda):=\theta(x, \lambda)+m_{ \pm}(\lambda) \phi(x, \lambda) ; \quad$ then $\quad \psi(\cdot, \lambda) \in L^{2}[a, b)\left(\lambda \in \mathbf{C}_{ \pm}\right)$

The Titchmarsh-Weyl $m(\lambda)$ function is defined by $m(\lambda)=m_{ \pm}(\lambda)$ $\left(\lambda \in \mathbf{C}_{ \pm}\right)$. The initial conditions given in (2.1) lead to the Neumann $m$-function. If $\Psi(\cdot, \lambda)$ is a solution of (1.1) which belongs to $L^{2}[a, b)$ then

$$
m(\lambda)=-\frac{\Psi(a, \lambda)}{\Psi^{\prime}(a, \lambda)}
$$

For further details of $m(\lambda)$ see Evans and Everitt [9], Everitt and Bennewitz [3] and the references contained therein. We use the notation of Evans and Everitt [9] and write $\lambda=r \exp (\mathrm{i} \theta)$, where $r \in(0, \infty)$, $\theta \in[0,2 \pi]$ and define the line segments

$$
\begin{aligned}
& L_{+}(\theta)=\{r \exp (\mathrm{i} \theta): r \in(0, \infty)\} \\
& L_{-}(\theta)=\{r \exp (\mathrm{i}(\theta+\pi)): r \in(0, \infty) \text { for } \theta \in(0, \pi / 2]\}
\end{aligned}
$$




$$
\begin{aligned}
\theta_{ \pm}= & \inf \{\theta \in(0, \pi / 2]: \text { for all } \phi \in[\theta, \pi / 2] \\
& \left.\mp \operatorname{Im}\left[\lambda^{2} m_{ \pm}(\lambda)\right] \geq 0\left(\lambda \in \mathrm{L}_{ \pm}(\phi)\right)\right\} \\
\theta_{0}= & \max \left(\theta_{+}, \theta_{-}\right)
\end{aligned}
$$

In Evans and Everitt [9, Section 6], it was proved that if (1.1) is SLP at $b$ and regular at $a$ then $\theta_{0} \neq 0$ and there is a valid inequality (1.2), (i.e. a finite $K$ ) if and only if $\theta_{0} \neq \frac{1}{2} \pi$, in which case the best constant $K=\sec ^{2} \theta_{0}$. Furthermore, with $r=|\lambda|$, and

$$
\begin{aligned}
E_{ \pm}= & \left\{r \in(0, \infty): \lambda \in L_{ \pm}\left(\theta_{0}\right) \text { and } \operatorname{Im}\left[\lambda^{2} m_{ \pm}(\lambda)\right]=0\right\} \\
& Y_{ \pm}(x, r)=\operatorname{Im}\left[\lambda \psi_{ \pm}(x, \lambda)\right], \quad\left(\lambda \in \mathrm{L}_{ \pm}\left(\theta_{0}\right)\right),
\end{aligned}
$$

then with $\theta_{0} \neq \frac{1}{2} \pi$ and $K=\sec ^{2} \theta_{0}$, the non-trivial functions $f$ which give equality in (1.2) fall into one of the following two distinct categories:

(i) $M f=0, f \in L^{2}[a, b)$ and either $f(0)=0$ or $f^{\prime}(0)=0$;

(ii) $E_{+} \cup E_{-} \neq \emptyset$ and $f(x)=A Y_{ \pm}(x, r)\left(r \in E_{ \pm}\right)$, with $A \in \mathbf{C}, A \neq 0$.

For further details see Evans and Everitt [9].

Examples of a valid inequality (1.2) have been obtained for $q(x)$ powers of $x$; for a full account of all the known examples that have been investigated analytically see Evans and Everitt $[9,10]$, Kirby [14] and Benammar et al. [2]. In all the examples that have been resolved an explicit expression has been available for $m(\lambda)$. Even then the analytic problems encountered when analysing $\operatorname{Im}\left[\lambda^{2} m(\lambda)\right]$ can be formidable and these difficulties are further compounded when $m(\lambda)$ is a meromorphic function of $\lambda$; see Evans et al. [11]. In this case it is shown in Everitt [12, Section 16] that a valid inequality is obtained if, and only if, $q(x)$ is replaced by $q(x)-\tau$, where $\tau$ is an eigenvalue of (1.1) with either Neumann or Dirichlet boundary conditions at the regular end-point $a$; the Neumann eigenvalues are the poles of $m(\lambda)$ and the Dirichlet eigenvalues its zeros. It is not surprising in view of the above remarks that when $p(x)=w(x)=1$ and $q(x)=x^{\alpha}$, the only case when the best constant has been determined analytically is when $\alpha=1$ and $\alpha=2$. In these examples the Neumann and Dirichlet eigenvalues are known and can be written explicitly and the corresponding $m(\lambda)$ function can also be written explicitly. Since information on the HELP inequality is so intractable analytically, recent efforts have been focused on developing 
numerical methods to obtain accurate estimates of the best constants $K$. There numerical algorithms have also been used to extract general properties of the HELP inequality. Success in this direction using these techniques has been reported in Brown et al. [4,5] and in Kirby [14]. In this paper we examine the case when $q(x)$ is periodic. For certain values of $\lambda$ the algorithm previously used to estimate the Titchmarsh-Weyl $m$-function can be very inefficient (see Section 3 ). Hence we discuss a new method for finding the $m$-coefficient which uses the property that when $q(x)$ is periodic we have special types of solutions called Floquet solutions (see Eastham [8]). We display our results using a graphical representation of the sets $\left\{\lambda: \mp \operatorname{Im}\left[\lambda^{2} m(\lambda)\right]=0\right\}$ in the first and third quadrant of $\mathbf{C}$ and these results can be found in Section 5.

\section{THE ALGORITHM}

In [4] and [5] there is a discussion on the computation of $m(\lambda)$ using an algorithm based on a "nesting circle" approach discussed in [1]. However it is shown in [4] that this algorithm works well when $q(x) \rightarrow \infty$ as $x \rightarrow \infty$; for example, when $q(x)=x^{\alpha}, \alpha>0$. For further details of the algorithm and implementation see Brown et al. [4, Section 2] and Kirby [14, Chapter 2]. However, the algorithm can be computationally expensive if the underlying solution of (1.1) is oscillatory. For example, when $q(x)=-x$, JWKB-type approximations reveal that the solutions to (1.1) are highly oscillatory and special methods have been developed for these problems which are discussed in [6]. Even when $q(x)$ is a periodic function this problem still arises when we look at $\lambda$ values which have small imaginary part and hence the method of [6] is not applicable. Thus we are forced to find a new algorithm to compute $m(\lambda)$. In order to do this we exploit the special type of solutions arising from such problems called Floquet solutions.

\subsection{Floquet Solutions}

A special type of problem arises in connection with differential equations of the form

$$
-y^{\prime \prime}+q(x) y=\lambda y
$$


where $q(x)$ is a periodic function with $q(x+\alpha)=q(x)$, and $\alpha$ is the period of the function $q(x)$. These are precisely the functions $q(x)$ outlined in Section 1 that we wish to examine. As outlined previously, the algorithms used to find $m(\lambda)$ in such cases are computationally expensive. We now give a basic outline of Floquet theory and describe how the $m(\lambda)$ function can be computed.

The differential equation (3.1) need not necessarily have periodic solutions, but it always possesses solutions of a special type. In fact, if

$$
\begin{gathered}
y(\alpha)=k y(0), \\
y^{\prime}(\alpha)=k y^{\prime}(0),
\end{gathered}
$$

where $k$ is a constant, then for all $x$,

$$
y(x+\alpha)=k y(x) .
$$

This is called a Floquet solution.

Let the solutions $\theta(x)$ and $\phi(x)$ be linearly independent solutions of (3.1) and satisfy the boundary conditions (2.1), then the Wronskian, $W(\theta, \phi)=1$ and there exists constants $A_{i j}, i, j=1,2$ such that

$$
\begin{aligned}
& \theta(x+\alpha)=A_{11} \theta(x)+A_{12} \phi(x), \\
& \phi(x+\alpha)=A_{21} \theta(x)+A_{22} \phi(x) .
\end{aligned}
$$

Every solution $\psi$ has the form

$$
\psi(x)=c_{1} \theta(x)+c_{2} \phi(x) .
$$

Now,

$$
\begin{aligned}
\psi(x+\alpha) & =\rho \psi(x)=c_{1} A_{11} \theta(x)+c_{1} A_{12} \phi(x)+c_{2} A_{21} \theta(x)+c_{2} A_{22} \phi(x) \\
& =\rho c_{1} \theta(x)+\rho c_{2} \phi(x),
\end{aligned}
$$

for some constant $\rho$ and so we obtain the matrix equation

$$
\left(\begin{array}{cc}
A_{11}-\rho & A_{21} \\
A_{12} & A_{22}-\rho
\end{array}\right)\left(\begin{array}{l}
c_{1} \\
c_{2}
\end{array}\right)=\left(\begin{array}{c}
\theta(x) \\
\phi(x)
\end{array}\right)
$$


These equations are satisfied by $c_{1}$ and $c_{2}$ both not zero if $\rho$ is such that

$$
\rho^{2}-\left(A_{11}+A_{22}\right) \rho+\operatorname{det} A=0 .
$$

In this paper we only consider the interval $[0, \infty)$ and using the boundary conditions in (2.1) it follows that

$$
\begin{aligned}
\theta(\alpha) & =-A_{12}, \\
\phi(\alpha) & =-A_{22} \\
\theta^{\prime}(\alpha) & =A_{11}, \\
\phi^{\prime}(\alpha) & =A_{21} .
\end{aligned}
$$

Thus, $\operatorname{det} A=W(\theta, \phi)(\alpha)=1$ and we have the following quadratic for $\rho$

$$
\rho^{2}-\left(\theta^{\prime}(\alpha)-\phi(\alpha)\right) \rho+1=0 .
$$

The expression $\theta^{\prime}(\alpha)-\phi(\alpha)$ is called the discriminant $D(\lambda)$. That is,

$$
D(\lambda)=\theta^{\prime}(\alpha)-\phi(\alpha)
$$

The discriminant function above differs from the one in Eastham $[8$, Section 1,2] as we have different boundary conditions. For a fuller account of the discriminant we refer the reader to Eastham's book.

\subsection{The $m$-Function}

We now consider the Neumann $m$-function introduced in Section 2 associated with the differential equation (3.1). The Dirichlet $m$-function is discussed in Titchmarsh [15, Section 11, p. 291]. We only give brief details below and refer the reader to the above reference.

Using the boundary conditions for $\theta$ and $\phi$ defined in the last subsection, we now look at the dependence of the $m$-function on $\theta$ and $\phi$ (evaluated at $\alpha$ ) and viewed as a function of the complex spectral function $\lambda$ in order to satisfy the boundary condition $\psi=-k \psi^{\prime}$. Substituting for $\psi=\theta+m \phi$ (see preliminaries, Section 2), we have

$$
m\left(\theta^{\prime}+m \phi^{\prime}\right)=-(\theta+m \phi) .
$$


Expressing the above as a quadratic in $m$, we obtain

$$
m^{2} \phi^{\prime}+m\left(\frac{\phi+\theta^{\prime}}{\phi^{\prime}}\right)+\frac{\theta}{\phi^{\prime}}=0,
$$

from which we obtain

$$
m=-\frac{\phi+\theta^{\prime}}{2 \phi^{\prime}} \pm \frac{1}{2 \phi^{\prime}} \sqrt{\left(\theta^{\prime}-\phi\right)^{2}-4}
$$

We can see from (3.3) and (3.2) that the discriminant and the $m$-function are connected. The values of $\lambda$ where $D(\lambda)<2$ correspond to the instability intervals and the values when $D(\lambda)>2$ correspond to the stability intervals (see [8]). As $\operatorname{Im}(\lambda) \rightarrow 0$ the imaginary parts of the solutions $\theta$ and $\phi$ and their derivatives tend to zero. Thus, the value of $m(\lambda)$ will tend to a real number if in the expression (3.3) the value of $\left(\theta^{\prime}-\phi\right)^{2}>4$. If this is the case then from the definition of $\theta_{ \pm}$in Section 2 we have that the smallest argument of $\lambda$ for which $\operatorname{Im}\left[\lambda^{2} m(\lambda)\right] \geq 0$ is $\pi / 2$ and so $\theta_{ \pm}=\pi / 2$ and thus there can be no valid inequality. Thus, we can only have valid inequalities if $\left(\theta^{\prime}-\phi\right)^{2}<4$ which corresponds to $\lambda$ lying in the instability intervals.

\subsection{Calculation of the Best Constant}

We have outlined the algorithm for finding $m(\lambda)$ and we now turn to the problem of estimating $\theta_{0}$ and hence evaluating the best constant $K$.

Using the result in Evans and Everitt [9, Corollary 7] it is shown in Brown et al. [4, Section 2.2] that the following is an equivalent definition for Eq. (2.3)

$$
\theta_{ \pm}=\sup \left\{\theta \in(0, \pi / 2]: \operatorname{Im}\left[\lambda^{2} m(\lambda)\right]=0 \text { for some } \lambda \in \mathrm{L}_{ \pm}(\theta)\right\}
$$

Thus $\theta_{0}$ can be determined by solving $\operatorname{Im}\left[\lambda^{2} m(\lambda)\right]=0$ for all $|\lambda|>0$ and using $\theta_{0}=\max \left(\theta_{+}, \theta_{-}\right)$; whence $K$ can be evaluated using $K=\sec ^{2}\left(\theta_{0}\right)$. To solve $\operatorname{Im}\left[\lambda^{2} m(\lambda)\right]=0$ we use an algorithm based on the NewtonRaphson method which tries to estimate $\partial m / \partial \lambda$, and in the cases when this fails, we resort to the Bisection method. 


\section{LOCATION OF THE SPECTRAL BANDS}

\subsection{Stability and Instability Intervals}

We consider the periodic problem

$$
-y^{\prime \prime}+q y=\lambda y, \quad y(a)=y(0), \quad y^{\prime}(a)=y^{\prime}(0)
$$

and label the eigenvalues $\lambda$ such that

$$
\lambda_{0} \leq \lambda_{1} \leq \lambda_{2} \leq \cdots \text { and } \lambda_{n} \rightarrow \infty \text { as } n \rightarrow \infty .
$$

Then, we consider the semi-periodic problem, that is with the boundary conditions

$$
y(a)=-y(0), \quad y^{\prime}(a)=-y^{\prime}(0)
$$

and label the eigenvalues $\mu$ by

$$
\mu_{0} \leq \mu_{1} \leq \mu_{2} \leq \cdots \text { and } \mu_{n} \rightarrow \infty \text { as } n \rightarrow \infty .
$$

Then the instability intervals, that is where $D(\lambda)<2$ (see $[8$, Theorem 2.3.1]) are given by

$$
\left[\lambda_{2 m}, \mu_{2 m}\right] \text { and }\left[\mu_{2 m+1}, \lambda_{2 m+1}\right] \text {, }
$$

where the numbers $\lambda_{n}$ and $\mu_{n}$ occur in the order

$$
\lambda_{0}<\mu_{0} \leq \mu_{1}<\lambda_{1} \leq \lambda_{2}<\mu_{2} \leq \mu_{3}<\lambda_{3} \leq \lambda_{4}<\cdots .
$$

Thus numerical estimates for $\lambda_{j}$ and $\mu_{j}$ may then be used to estimate the location of the instability intervals. We are now in a position to locate the bands by numerically computing the eigenvalues of the periodic and semi-periodic problem.

\subsection{The Rayleigh-Ritz method}

To estimate numerically the ends of the bands, that is, the eigenvalues of both the periodic and semi-periodic problem, we have used the Rayleigh-Ritz method. 
It can be shown that the appropriate variational equation is

$$
\int_{0}^{\pi / 2}\left(\phi_{i}^{\prime} \phi_{j}^{\prime}+q(x) \phi_{i} \phi_{j}\right) \mathrm{d} x=\int_{0}^{\pi / 2} \phi_{i} \phi_{j} \mathrm{~d} x
$$

where $q(x)$ is either $\pm \sin (x), \pm \cos (x)$ or the constant periodic problem (see Section 4.3). We write $k_{i j}=\int_{0}^{\pi / 2}\left(\phi_{i}^{\prime} \phi_{j}^{\prime}+q(x) \phi_{i} \phi_{j}\right) \mathrm{d} x$ and $m_{i j}=$ $\int_{0}^{\pi / 2} \phi_{i} \phi_{j} \mathrm{~d} x$. For the symmetric (periodic) problem we choose suitable basis functions from which to construct our approximations to the solution. In this case a suitable linearly independent basis is

$$
\{1, \sin (i x), \cos (i x), \ldots, i=1, \ldots, n\} .
$$

For the skew-symmetric (semi-periodic) problem we choose

$$
\{\sin ((1 / 2-i) x), \cos ((1 / 2-i) x), \ldots,: i=1, \ldots, n\}
$$

and we construct an approximate solution $u_{n}(x)$ in the form

$$
u_{n}(x)=\sum_{i=1}^{n} c_{i} \phi_{i}(x)
$$

We obtain approximations (in fact we obtain upper bounds if the arithmetic were done exactly) to the eigenvalues when we solve the generalized matrix eigenvalue problem

$$
K U=\lambda M U,
$$

where $U=\left(c_{1}, \ldots, c_{n}\right)$ and $K=k_{i j}, M=m_{i j}$. This procedure is easily implemented using the symbolic computer algebra system Maple, which is able to handle the problem of integrating and differentiating these functions as well as solving the eigenvalue problem.

\subsection{Location of the Bands for the Constant Case}

In this paper we shall refer to the "constant-periodic" case as being the following problem:

$$
q(x)=\left\{\begin{array}{ll}
-1, & x \in[(2 i-2) \pi,(2 i-1) \pi] \\
+1, & x \in[(2 i-1) \pi, 2 i \pi]
\end{array} i=1,2, \ldots\right.
$$

The Rayleigh-Ritz method was used with $n=20$ and the values of $\lambda_{n}$ obtained from the method for the periodic problem were as 
follows: $-0.526958,0.560950,2.261250,2.463253,6.186753,6.391791$, $12.188365,12.352148$ and 20.195896. Whilst for the semi-periodic problem the $\mu_{n}$ we obtained were: $-0.546610,0.766004,1.466529$, $3.948054,4.170129,8.974719,9.080333,15.985476$ and 16.046021 . The values quoted above are correct to all places of decimals. Then the bands can be determined by appropriately combining the results using the information in Section 4.1. Thus the bands lie between each pair of values given below (truncated to 3 decimal places).

$$
\begin{aligned}
& (-0.546,-0.526), \quad(0.560,0.766), \quad(1.466,2.261), \\
& (2.463,3.948), \quad(4.170,6.186), \quad(6.391,8.974), \\
& (9.080,12.188), \quad(12.352,15.985), \quad(16.046,20.195) .
\end{aligned}
$$

\subsection{Location of the Eigenvalues, the Constant Case}

Below we give the first few eigenvalues of (1.1) with Neumann or Dirichlet boundary conditions imposed at the regular end-point. In order to deduce the eigenvalues of Neumann and Dirichlet type we needed to examine the analytic expression for the $m$-function and from its real and imaginary parts, deduce its zeros and poles. We have shown the imaginary part of the $m$-function in Fig. 2.

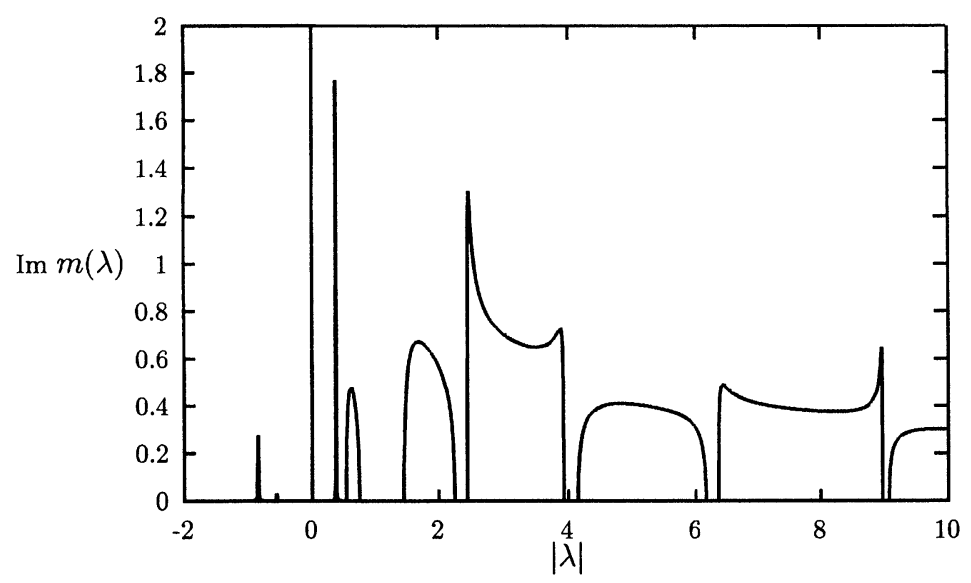

FIGURE $2|\lambda|$ plotted against $\operatorname{Im} m(\lambda)$. 
Our numerical evidence suggests that there are only 2 poles, that is, eigenvalues with Neumann conditions imposed at the regular end-point and that there may be infinitely many zeros located beween the spectral bands.

\subsection{Some Asymptotic Results for the $m$-Function in the Constant- Periodic Case}

In this case the solutions to (1.1) can be found explicitly and computed numerically. In view of the theoretical results in Section 3.1 we need to only evaluate the solutions to the second-order differential equation with $q(x)$ defined in (4.1) at the point $2 \pi$. Let $\alpha=\sqrt{-1-\lambda}$ and $\beta=\sqrt{1-\lambda}$. Then by considering the $\theta$ and $\phi$ solutions to (3.1) over the intervals $[0, \pi]$ and $[\pi, 2 \pi]$ (using the boundary conditions) we obtain the following solutions at $2 \pi$

$$
\left.\begin{array}{rl}
\phi & =(-1 / 2-\alpha / 2 \beta) \cosh (\alpha+\beta) \pi+(-1 / 2+\alpha / 2 \beta) \cosh (\alpha-\beta) \pi \\
\phi^{\prime} & =(-\beta / 2-\alpha / 2) \sinh (\alpha+\beta) \pi-(-\beta / 2+\alpha / 2) \sinh (\alpha-\beta) \pi \\
\theta & =(1 / 2 \alpha+1 / 2 \beta) \sinh (\alpha+\beta) \pi-(1 / 2 \beta+1 / 2 \alpha) \sinh (\alpha-\beta) \pi \\
\theta^{\prime} & =(\beta / 2 \alpha+1 / 2) \cosh (\alpha+\beta) \pi+(1 / 2-\beta / 2 \alpha) \cosh (\alpha-\beta) \pi
\end{array}\right\} .
$$

From (3.3) we note that the expression for the $m$-function involves either taking the positive square root or the negative square root. In order to choose the correct $m$-function we have to ensure that the following result holds

$$
\frac{\operatorname{Im} m(\lambda)}{\operatorname{Im} \lambda}>0
$$

See [7]. In this section we analyse the HELP inequality for the constantperiodic case using the form of the $m$-function as derived in the previous section. We then display our numerical results in light of our analytic results.

Using the results in (4.2), we can analyse the $m$-function in the limit as $\lambda \rightarrow 0$ and the translate $\tau=0$. Then as $\lambda \rightarrow 0$ we have $\alpha \rightarrow \mathrm{i}$ and $\beta \rightarrow 1$. Then it can be shown that in this limit $\phi+\theta^{\prime} \rightarrow 0$ and $\phi^{\prime} \rightarrow \sinh \pi$. 
Then the term $\sqrt{\left(\theta^{\prime}-\phi\right)^{2}-4} \rightarrow 2 \sinh \pi$. Thus the $m$-function tends to either 1 or -1 as $\lambda \rightarrow 0$.

For (4.3) to hold we need to examine the sign of the $m$-function. We do this by taking a Taylor series expansion about $\lambda=0$. We set $\alpha=\mathrm{i}(1+\lambda / 2)$ and similarly $\beta=1-\lambda / 2$ and find that when $\operatorname{Im} \lambda>0$ we must take the + sign and the - sign when $\operatorname{Im} \lambda<0$ to obtain

$$
\lim _{\lambda \rightarrow 0} m(\lambda)=1 .
$$

Thus using the definitions of $\theta_{ \pm}$and hence $\theta_{0}$ in Section 2 we have that $\theta_{-}=0$ and $\theta_{+}=\pi / 2$. That is, there is no valid inequality when $\tau=0$. This is confirmed by our numerical calculations shown in Section 5 .

As $\lambda \rightarrow \infty$ we have that $\alpha \rightarrow \mathrm{i} \sqrt{\lambda}$ and $\beta \rightarrow \mathrm{i} \sqrt{\lambda}$. Thus, we obtain the approximations

$$
\begin{aligned}
& \phi \approx-\cos (2 \sqrt{\lambda} \pi), \\
& \phi^{\prime} \approx \sqrt{\lambda} \sin (2 \sqrt{\lambda} \pi), \\
& \theta^{\prime} \approx \cos (2 \sqrt{\lambda} \pi) .
\end{aligned}
$$

Substituting these terms in the expression for the $m$-function we obtain

$$
\lim _{|\lambda| \rightarrow \infty} m(\lambda)=\frac{i}{\sqrt{\lambda}}
$$

(see [13] on asymptotic behaviour of the $m$-function.) Once again the sign outside the root needs to be chosen correctly so that the $m$-function satisfies (4.3). Thus we see from Section 2, that with this result we have for $\lambda$ in the third quadrant, $\lim _{|\lambda| \rightarrow \infty} \theta=0$ and for $\lambda$ in the first quadrant $\lim _{|\lambda| \rightarrow \infty} \theta=\pi / 3$.

\section{RESULTS}

Where possible we have compared our results with analytic results obtained from the solutions in closed form, the original algorithm and the new method which is based on using the properties of the Floquet solutions. 


\subsection{Translates for the Bands for the Constant Case}

We first consider the constant case and we have chosen to take a translate from approximately the centre of each band. In Figs. 3 and 4 we display the results from solving $\operatorname{Im}\left[\lambda^{2} m(\lambda)\right]=0$ for $\lambda$ in the first and third quadrants. In all the cases for $\theta_{+}$the curves are always less than $\pi / 3$ and this is attained at infinity.

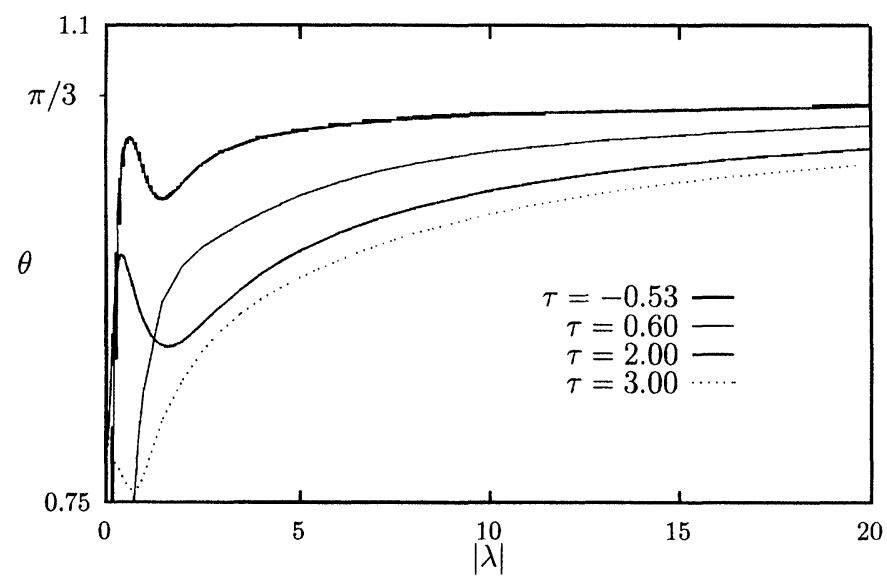

FIGURE 3 Graph of $\operatorname{Im}\left[\lambda^{2} m(\lambda)\right]=0$ when $\lambda \in L_{+}$for the case $q$ is periodic constant on $[0, \infty]$.

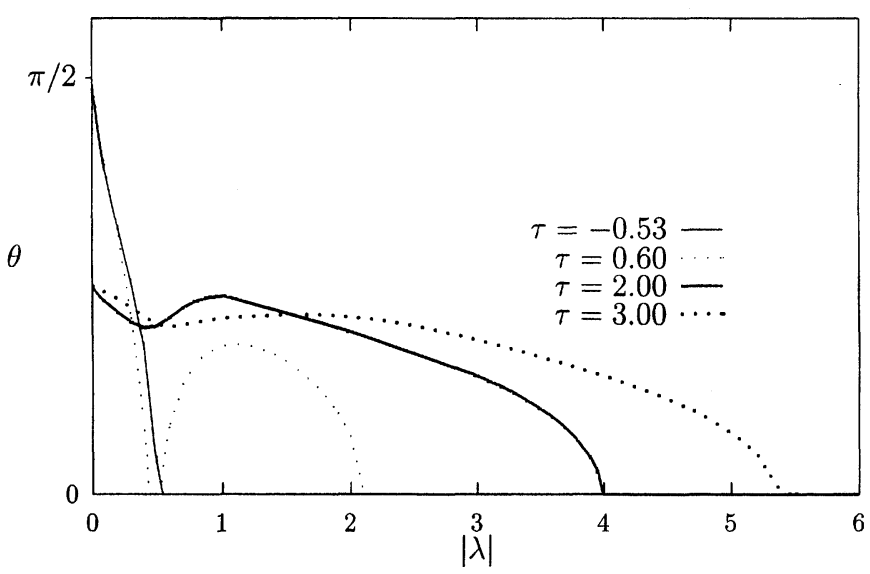

FIGURE 4 Graph of $\operatorname{Im}\left[\lambda^{2} m(\lambda)\right]=0$ when $\lambda \in L_{-}$for the case $q$ is periodic constant on $[0, \infty]$. 
However, for the $\theta_{-}$case, we have that for the -0.53 translate the value of $\theta_{-}$is approximately 1.556 and is attained at zero, and hence the value of the best constant is extremely large (approximately 4568). Similarly for the next translate the value of $\theta_{-}$is also large, 1.444 , but not as large as the first translate.

Here the best constant is approximately 62.5 . But for the remaining translates we find that the value of $\theta_{-}$is less that $\theta_{+}$and the best constant for these translates is 4 . We have also noted that the value of the best constant varies only slightly throughout the band and is largest at the ends of the bands.

\subsection{Neumann Eigenvalues}

We now consider the effect of translating the problem so that the pole of the $m$-function now lies at the origin. That is, we translate by $\tau_{p, 1}$, the first pole and $\tau_{p, 2}$, the second pole. These values can be found in Table I in the Neumann column.

The results are that the value of $\theta_{+}$is always less than $\pi / 3$ (attained at infinity) and the value of $\theta_{-}$for the first translate is zero, which can be seen in Figs. 5 and 6. For the second translate $\theta_{-}<\theta_{+}$.

\subsection{Dirichlet Eigenvalues}

We now examine what happens when we shift the problem so that there are zeros at the origin. We consider only the first seven translates. A curious feature of this example is that for the odd numbered translates (certainly $1,3,5$ and 7 ) the value of $\theta_{+}$is greater than $\pi / 3$. That is for all examples considered, the graph starts at $\pi / 3$ (see Figs. 7-10), reaches a maximum and then decreases to $\pi / 3$ as $\lambda \rightarrow \infty$. However for the even

TABLE I Eigenvalues for constant case

\begin{tabular}{lr}
\hline Neumann & \multicolumn{1}{c}{ Dirichlet } \\
\hline-0.834322457 & -0.348878870 \\
0.398428672 & 1.250000000 \\
& 2.272990582 \\
& 4.139909207 \\
6.229218608 \\
9.073354466 \\
& 12.234794510 \\
\hline
\end{tabular}




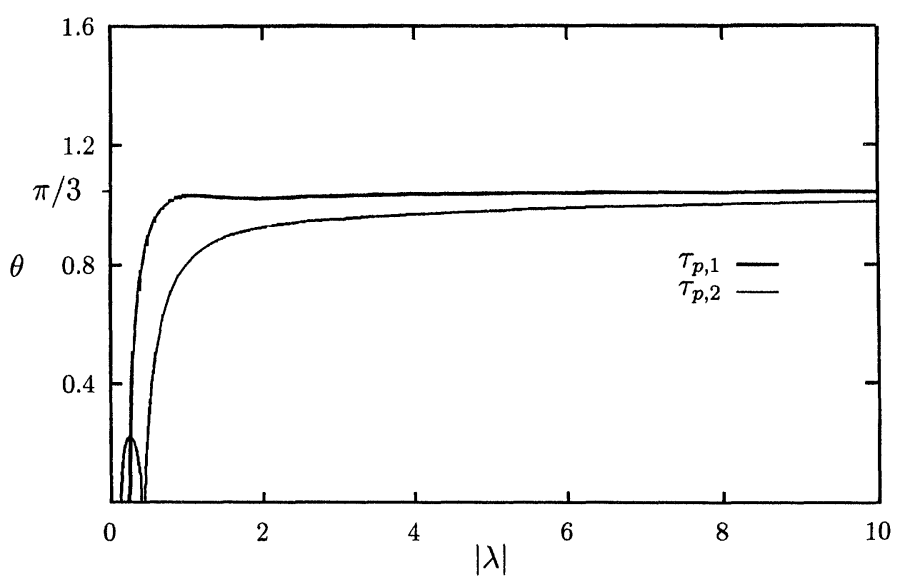

FIGURE 5 Graph of $\operatorname{Im}\left[\lambda^{2} m(\lambda)\right]=0$ when $\lambda \in L_{+}$for the case when we translate by the two poles.

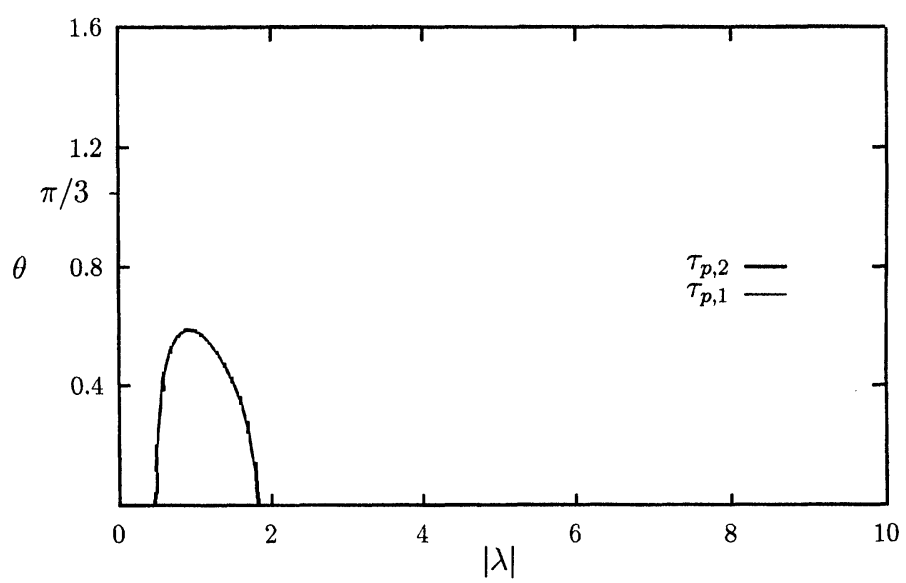

FIGURE 6 Graph of $\operatorname{Im}\left[\lambda^{2} m(\lambda)\right]=0$ when $\lambda \in L_{-}$for the constant case when we translate by the two poles.

numbered translates there is no peak and the value of $\theta_{+}$for these cases is always $\pi / 3$. For the $\theta_{-}$case, the odd numbered translates give a value of less than $\pi / 3$, this being attained at zero and infinity.

However for the even numbered translates, the roles are reversed and this time the value of $\theta_{-}$is greater than $\pi / 3$. In summary, for the odd 


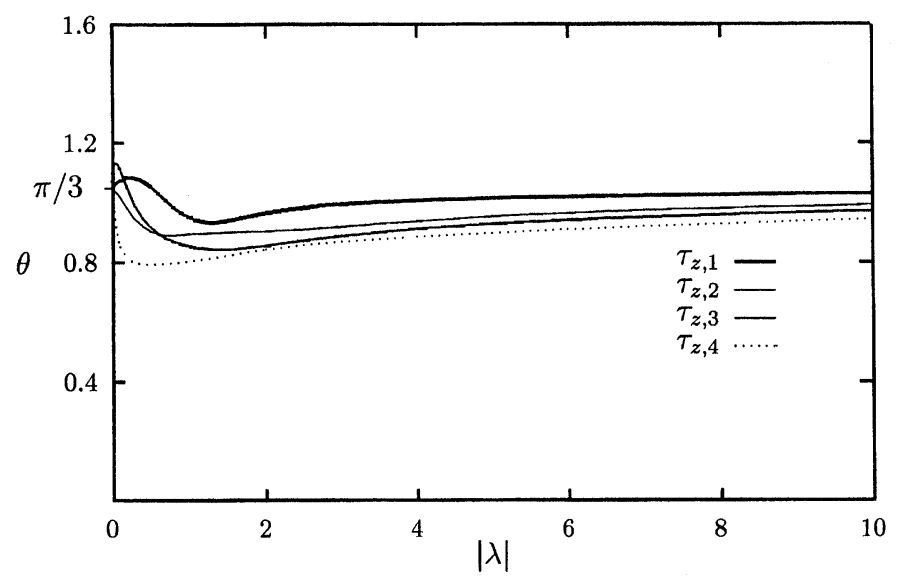

FIGURE 7 Graph of $\operatorname{Im}\left[\lambda^{2} m(\lambda)\right]=0$ when $\lambda \in L_{+}$for the case when we translate by the first four zeros.

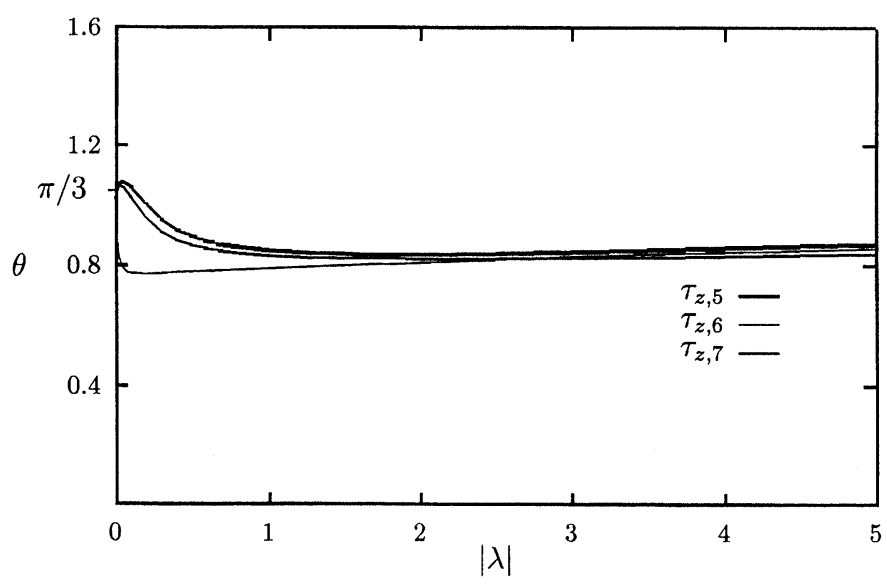

FIGURE 8 Graph of $\operatorname{Im}\left[\lambda^{2} m(\lambda)\right]=0$ when $\lambda \in L_{+}$for the case when we translate by the next three zeros.

numbered translates the value of $\theta_{0}$ can be found by examining the values of $\theta_{+}$and for the even numbered translates we look at the values of $\theta_{-}$. In Fig. 11 we show the portion of the graph when $|\lambda|$ is small. In Table II we give the values of the best constants for the first seven Dirichlet translates. 


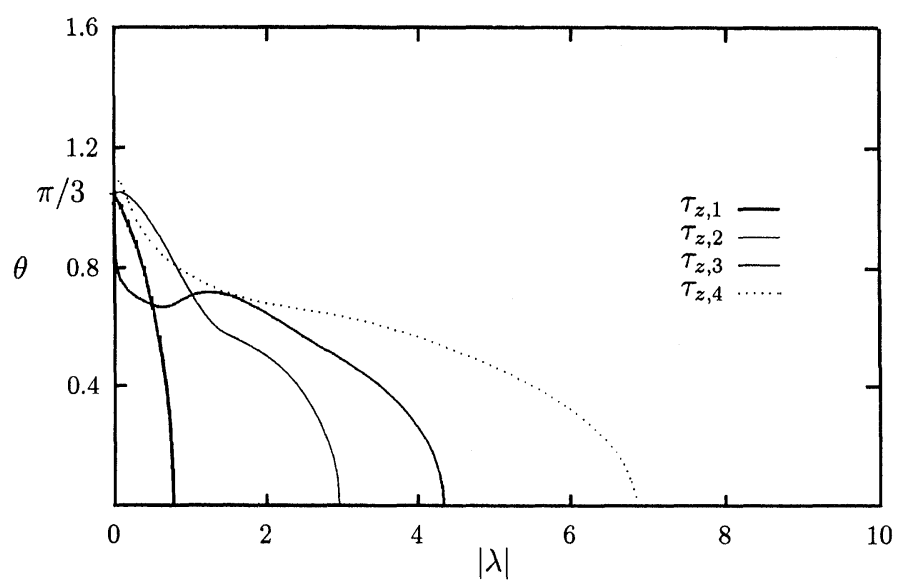

FIGURE 9 Graph of $\operatorname{Im}\left[\lambda^{2} m(\lambda)\right]=0$ when $\lambda \in L_{-}$for the case when we translate by the first four zeros.

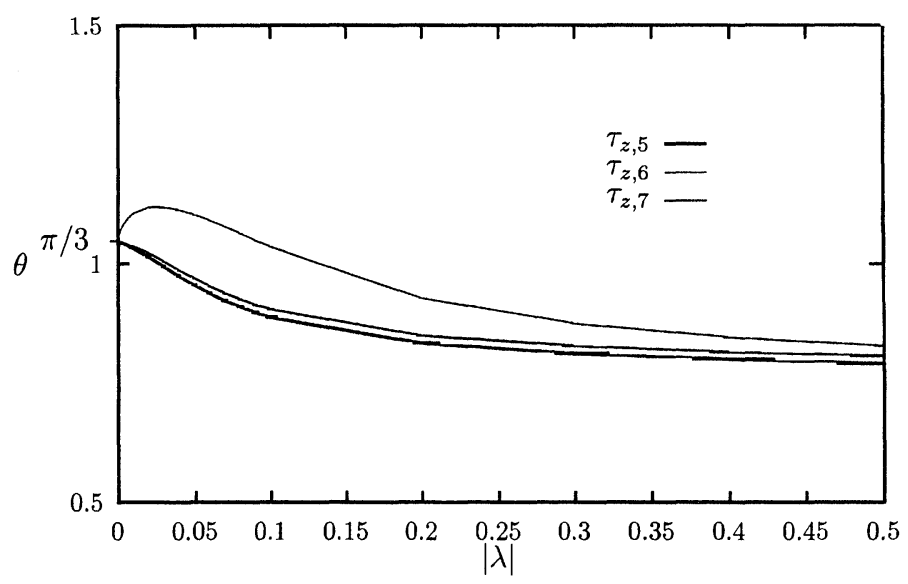

FIGURE 10 Graph of $\operatorname{Im}\left[\lambda^{2} m(\lambda)\right]=0$ when $\lambda \in L_{-}$with $x$-range restricted to show behaviour at origin.

\subsection{The Case $q(x)=\cos (x)$}

We now turn our attention to the case when $q(x)=\cos (x)$. For this example we do not know the exact form of the solutions and we need to estimate the solutions numerically. For similar reasons as for the 


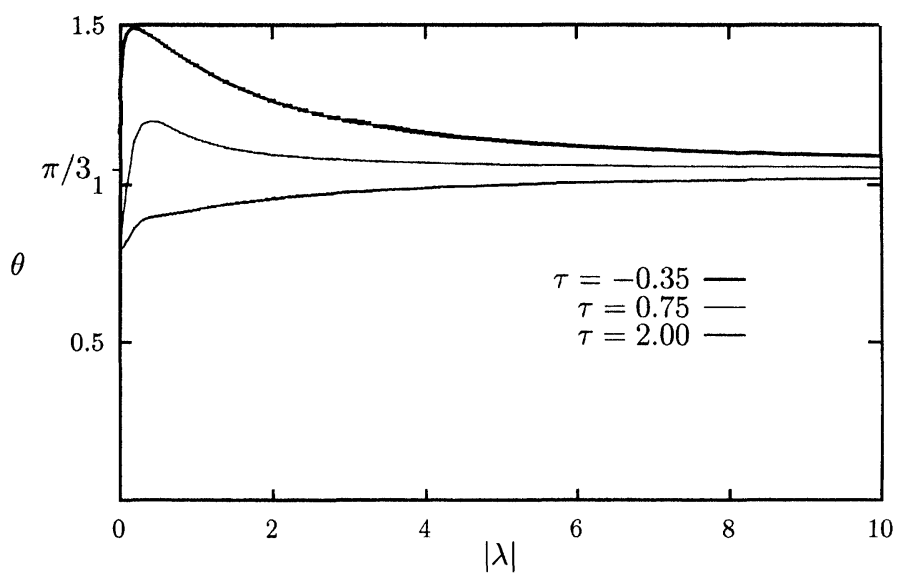

FIGURE 11 Graph of $\operatorname{Im}\left[\lambda^{2} m(\lambda)\right]=0$ when $\lambda \in L_{+}$for the case $q(x)=\cos (x)$ on $[0, \infty]$.

TABLE II Values of the best constants for various Dirichlet translates

\begin{tabular}{lccc}
\hline $\begin{array}{l}\tau_{z, n} \text { Dirichlet } \\
\text { translate } n\end{array}$ & $\begin{array}{c}E_{ \pm} \\
\text {(estimated peak) }\end{array}$ & $\theta_{0}$ & $\begin{array}{c}K \\
\text { (best constant) }\end{array}$ \\
\hline 1 & 0.236 & 1.083188 & 4.55569 \\
2 & 0.079 & 1.053413 & 4.08769 \\
3 & 0.053 & 1.131975 & 5.53965 \\
4 & 0.056 & 1.090910 & 4.69160 \\
5 & 0.049 & 1.074122 & 4.40419 \\
6 & 0.025 & 1.118885 & 5.24399 \\
7 & 0.032 & 1.061043 & 4.19979 \\
\hline
\end{tabular}

constant case the original algorithm discussed in [4] and [5] is slow and inefficient.

We will need to find the eigenvalues (if any) which lie in the gaps. The spectral bands once again will be computed using the Rayleigh-Ritz method. The results for $q=\sin x$ and $q=\cos x$ are the same, since both problems have the same essential spectrum. The bands are $(-0.37848922,-0.3476691),(0.59479997,0.91805813),(1.29316628$, 2.28515693), (2.34258062, 4.03192190) and (4.03530094, 6.27083725).

We used the code SLEIGN2 to obtain the eigenvalues for the $\cos (x)$ and $\sin (x)$ problems over $[0, \infty)$. In using SLEIGN2 we approximated the singular problem over $[0, \infty)$ by a regular one over a finite interval, taking the right-hand end-point as a multiple of $\pi$. We found no 
eigenvalues within the gaps for the case when $q(x)=\cos (x)$. But for $q(x)=\sin (x)$, we found an eigenvalue in each gap. In Fig. 11 we show the graphs when we take a translate in each of the first three bands. The best constant varies only a little as we traverse the band and we only give the results for $q=\cos x$.

Dirichlet boundary conditions were then imposed at both end-points. Output from the code revealed eigenvalues which clustered inside the known location of the bands. Those lying outside were then taken to be eigenvalues. Increasing the right-hand end-point then forced more eigenvalues into the known region of the bands and hopefully the values for the eigenvalues were converging to some more accurate value for the eigenvalues of the singular problem. For the problem $q(x)=\sin (x)$ the first three eigenvalues are approximately $-0.183389680,1.20426619$, 2.33440089 . We suspect that only the first seven digits are correct. The values of $b=40 \pi$ and a tolerance of $10^{-8}$ was used. Similarly, the Neumann eigenvalues are $-0.533708096,0.992769778$ and 2.29900837 approximately. It is unclear whether these are eigenvalues since we are surprised that these examples do not produce a best constant. In all the examples we have analysed to date, this has always been the case and so we are suspicious.

For the case $q(x)=\cos (x)$ we have also looked at the value of the best constant along the first two bands. The results are that the best constant varies very little across the band. For example in the first band for the following translates: $-0.35,-0.36,-0.37,-0.377$ the values of the best constants are $152.81,152.30,152.65$ and 153.39 respectively. Similar results are available for the second band, where we considered the following translates: $0.6,0.7,0.8,0.9$ where the best constants are $8.58,7.76,7.53,9.00$. It can be seen that the values of the best constant increases as we approach the ends of the bands.

\section{References}

[1] F.V. Atkinson, On the location of the Weyl circles. Proc. Roy. Soc. Edinb. A 88 (1981), 345-356.

[2] M. Benammar, C. Bennewitz, M.J. Beynon, B.M. Brown, N.G.J. Dias, W.D. Evans, W.N. Everitt, V.G. Kirby and L.L. Littlejohn, A catalogue of HELP and Help-type integral and series inequalities. Recent progress in Inequalities (Vol. 1). International Memorial Conference, D.S. Mitrinovic (1996).

[3] C. Bennewitz and W.N. Everitt, Some remarks on the Titchmarsh-Weyl m-coefficient. A Tribute to Ake Pleijel (Proceedings of the Pleijel Conference, Uppsala, 1979) pp. 49-108. Uppsala, Sweden: Department of Mathematics, University of Uppsala. 
[4] B.M. Brown, V.G. Kirby and J.D. Pryce, Numerical determination of the TitchmarshWeyl $m$-coefficient and its applications to HELP inequalities. Proc. Roy. Soc. Lond. (A) 426 (1989), 167-188.

[5] B.M. Brown, V.G. Kirby and J.D. Pryce, A numerical method for the determination of the Titchmarsh-Weyl m-coefficient. Proc. Roy. Soc. Lond (A) 435 (1991), 535-549.

[6] B.M. Brown, M.S.P. Eastham, W.D. Evans and V.G. Kirby, Repeated diagonalization and the numerical computation of the Titchmarsh-Weyl $m(\lambda)$-function. Proc. Roy. Soc. Lond. (A) 445 (1994), 113-126.

[7] E.A. Coddington and N. Levinson, Theory of Ordinary Differential Equations. McGraw-Hill (New York) (1955).

[8] M.S.P. Eastham, The Spectral Theory of Periodic Differential Equations. Scottish Academic Press, Edinburgh and London (1973).

[9] W.D. Evans and W.N. Everitt, A return to the Hardy-Littlewood inequality. Proc. Roy. Soc. Lond. (A). 380 (1982), 447-486.

[10] W.D. Evans and W.N. Everitt, Hardy-Littlewood integral inequalities. Lecture Notes in Pure and Applied Mathematics. Inequalities; fifty years on from Hardy, Littlewood and Polya, Vol. 129, (1991).

[11] W.D. Evans, W.N. Everitt, W.K. Hayman and S. Ruscheweyh, On a class of integral inequalities of Hardy-Littlewood type. J. d' Anal. Math. 46 (1986), 118-147.

[12] W.N. Everitt, On an extension to an integro-differential inequality of Hardy, Littlewood and Polya, Proc. Roy. Soc. Edin. A 69 (1971/72), 295-333.

[13] H.G. Kaper and M.K. Kwong, Asymptotics of the Titchmarsh-Weyl $m$-coefficient for integrable potentials. Proc. Roy. Soc. Edinb. A 103 (1986), 347-358.

[14] V.G. Kirby, A numerical method for determining the Titchmarsh-Weyl $m$-coefficient and its applications to certain integro-differential inequalities. Ph.D. Thesis, University of Wales College of Cardiff (1990).

[15] E.C. Titchmarsh, Eigenfunction Expansions, Part 1, 2nd edn. Oxford University Press (1962). 